\title{
The Value of 18 Fluorine-fluorodeoxyglucose Positron Emission Tomography/Computed Tomography Imaging in Breast Cancer Staging
}

\author{
Ahmed Tawakol ${ }^{1}$, Maha Khalii ${ }^{2 *}$, Yasser G. Abdelhafez ${ }^{2}$, Mai Hussein ${ }^{1}$, Mohamed Fouad Osman $^{3}$ \\ ${ }^{1}$ Department of Oncology and Nuclear Medicine (NEMROCK), Kasr El-Ainy Hospital, Cairo University, Egypt; ${ }^{2}$ Department of \\ Radiotherapy and Nuclear Medicine, South Egypt Cancer Institute, Assiut University, Egypt; ${ }^{3}$ Department of Radiology, Kasr \\ El-Ainy Hospital, Cairo University, Egypt
}

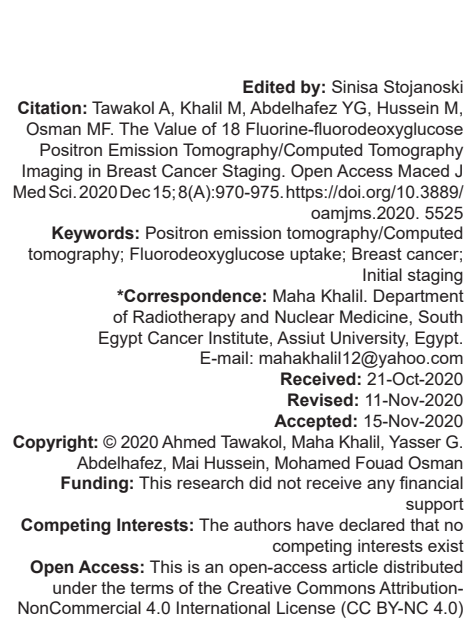

\section{Background}

Breast cancer remains the most commonly diagnosed cancer in women. It is the main cause of death from cancer in women in the world and affecting not only the elderly but also younger patients [1]. Accurate staging is important for deciding on therapy and defining the prognosis. 18 fluorinefluorodeoxyglucose $\left({ }^{18} \mathrm{~F}-\mathrm{FDG}\right)$ positron emission tomography/computed tomography (PET/CT) has the advantage of providing both functional and anatomic information compared with only the anatomic description provided by conventional imaging. ${ }^{18}$ F-FDG-PET has an important role in diagnostic staging, recurrence evaluation, and assessment the response to therapy [2]. ${ }^{18}$ F-FDG PET/CT fusion studies have the advantage of anatomic characterization in addition to the functional information provided by PET. Furthermore, it would be less likely to mistake the normal physiologic accumulation seen in some PET studies when no corresponding lesion is observed on the CT portion. An important advantage of ${ }^{18} \mathrm{~F}$ FDG PET/CT is its ability to depict malignant disease in lymph nodes that do not appear pathologically enlarged at CT and in the evaluation of regional lymph nodes, in particular, is its greater accuracy for detecting and locating metastases in the internal mammary and supraclavicular lymph nodes [3], [4]. PET is more efficient than CT or bone scintigraphy for depicting lytic or mixed bone metastases and bone marrow lesions. ${ }^{18}$ F-FDG PET could be more sensitive in detecting lytic metastases than sclerotic metastases; the latter lesions commonly show lowgrade uptake and less commonly appear completely devoid of any FDG activity [5]. Nonetheless, they show osteo-condensation on underlying CT images so that they can be efficiently detected by hybrid PET/ CT [6]. Many studies have shown that ${ }^{18}$ F-FDG PET/ $\mathrm{CT}$ is more accurate than the conventional methods in staging breast carcinoma, accordingly impacted the therapeutic management of the patients [6]. 
In this study, we aimed to evaluate the value of ${ }^{18} \mathrm{~F}-\mathrm{FDG}$ PET/CT in detecting distant lesions in patients with breast cancer who were assessed by conventional imaging methods and its impact in staging and changing therapeutic management.

\section{Methods}

The Institutional Review Board approved this prospective study. During the period from November 2017 to September 2019, 80 eligible patients were included in this study ( 1 male and 79 female; mean age $51 \pm 13$ years). The common eligibility criteria were a histopathologically confirmed diagnosis of breast cancer and pretherapy (staging) whole-body ${ }^{18} \mathrm{~F}-\mathrm{FDG}$ PET/CT study performed with contrast-enhanced CT (CE-CT). All the PET/CT studies were performed either at the Center of Clinical Oncology and Nuclear medicine (NEMROCK). However, other parts of the conventional work-up (mammography, breast ultrasound, and/or bone scans) were performed in outside institutions.

We excluded pregnant ladies and patients who had surgical excision of the tumor, previously received chemo or radiotherapy.

All patients underwent clinical assessment by an oncologist experienced in breast cancer management. The evaluation procedures included history, physical examination, mammography with/ without breast ultrasound, and CE-CT of neck, chest, abdomen, and pelvis. Mammography and breast ultrasound were performed in many outside institutions, according to their intuitional protocols. Whole-body CE-CT was part of the ${ }^{18} \mathrm{~F}-\mathrm{FDG}$ PET/CT acquisition.

\section{${ }^{18}$ F-FDG PET/CT acquisition and reconstruction protocol}

Patients were instructed for preparation according to the EANM procedure guidelines for ${ }^{18}$ F-FDG PET/CT tumor imaging: Version 2.0 [7]. Briefly, patients were required to fast for $6 \mathrm{~h}$ and asked to void before the examination. The patients were instructed to stay calm and avoid walking or any exercise before and after injection of ${ }^{18} \mathrm{~F}-\mathrm{FDG}$ to prevent physiologic muscle FDG uptake. Blood glucose level measured before injection and fasting levels were $<200 \mathrm{mg} / \mathrm{dL}$ (70-170 $\mathrm{mg} / \mathrm{dL}$ in our cohort). Warm environment was available before injection to avoid brown fat uptake.

A dose of $3.7-5.2 \mathrm{MBq} / \mathrm{Kg}$ of ${ }^{18} \mathrm{~F}-\mathrm{FDG}$ was injected through a hand or antecubital vein, contralateral to the breast lesion, or using foot veins in the presence of bilateral breast lesions. The injection was performed 45-90 min before the start of PET/CT acquisition. For opacification of bowel loops, 400-600 ml of contrast material diluted with water and swallowed $1 \mathrm{~h}$ before PET/CT imaging.

The scanner used in NEMROCK was Ingenuity TF 64 (Philips Healthcare, Cleveland, OH, USA), a PET/CT scanner combining a modular, LYSO-based PET component with a 64-channel CT component. The CT was based on the Ingenuity CT (Philips Healthcare).

Acquisition and reconstruction protocol was previously described [8]. Briefly, a low-dose noncontrast CT scan was performed first followed by a whole-body PET acquisition then a whole-body CE-CT scan.

Low-dose CT was acquired in a helical mode, using $120 \mathrm{kV}, 60 \mathrm{mAs}$, and a $512 \times 512$ matrix size, acquiring a field of view (FOV) of $700 \mathrm{~mm}$. This CT scan was used for attenuation correction.

PET scan was acquired in a three-dimensional mode over the same anatomical regions starting from the skull vertex to the level of the mid-thigh. The acquisition time was 2 min per bed position, in 9-bed positions. Reconstructed slice thickness was $5 \mathrm{~mm}$.

Immediately after completing PET acquisition, a diagnostic CT with contrast was acquired using $120 \mathrm{kV}, 300 \mathrm{mAs}$, and a $512 \times 512$ matrix size. The acquired FOV was $500 \mathrm{~mm}$ using dose automatic modulation in the $Z$ direction. Non-ionic contrast media were IV injected using automatic injector at a rate of $4 \mathrm{ml} / \mathrm{s}$, in a dose of $1-2 \mathrm{ml} / \mathrm{kg}$ (maximum $<150 \mathrm{ml}$ ). Slice thickness was $1.0 \mathrm{~mm}$.

The whole study took about 20-30 min Raw data were reconstructed using a standard manufacturer's iterative algorithm. Axial PET and CT images obtained and then reformatted into sagittal and coronal images to allow easier image interpretation.

\section{PET/CT and CE-CT interpretation}

Two nuclear medicine physicians with 7 and 13 years of experience, read the ${ }^{18} \mathrm{~F}-\mathrm{FDG}$ PET/CT study, together with the CE-CT images, but blinded to the radiological readings. CE-CT was independently and blindly read by an experienced radiologist (16-years' experience). A checklist for local tumor extension ( $T$ status), regional lymph node involvement ( $\mathrm{N}$ status), and distant lesions ( $\mathrm{M}$ status) was used for both modalities. The three components ( $\mathrm{T}, \mathrm{N}$, and $\mathrm{M}$ ) were collectively used to stage the patient according to the American Joint Committee on Cancer (AJCC) staging system, $8^{\text {th }}$ Edition.

On ${ }^{18}$ F-FDG PET/CT, lesions were considered positive if ${ }^{18} \mathrm{~F}$-FDG uptake was focally increased in the lesion and was greater than surrounding background tissues. Any discrepancy between the two readers was solved by consensus. 


\section{Evaluation of the impact}

Initial clinical staging was performed by the oncologist, according to conventional work-up, including the CE-CT reads, then, the treatment plans were decided using only this information. The results of ${ }^{18} \mathrm{~F}$ FDG-PET/CT were then revealed and any changes in the individual component of the $\mathrm{T}, \mathrm{N}$, and/or $\mathrm{M}$ states were recorded and a new treatment plan was discussed. Any change in management based on the new ${ }^{18} \mathrm{~F}-\mathrm{FDG}$ PET/CT findings was recorded.

\section{Statistical analysis}

Data were statistically described in terms of mean \pm standard deviation (SD), median and range, or frequencies (number of cases) and percentages as appropriate. Correlation was performed using Spearman rank correlation for ordinal and continuous variables with non-normal distributions. McNemar's test was used to measure the difference in proportions of disagreement between the two modalities $\left({ }^{18} \mathrm{~F}-\mathrm{FDG}\right.$ PET/CT and conventional work-up). Kappa coefficient used to measure the agreement between the two modalities. The level of agreement was categorized as poor $(\mathrm{k}<0)$, slight $(\mathrm{k}=0-0.20)$, fair $(\mathrm{k}=0.21-0.4)$, moderate $(\mathrm{k}=0.41-0.6)$, substantial $(\mathrm{k}=0.61-0.8)$, and perfect agreement $(\mathrm{k}=0.81-1.0)$ [9]. All analyses were performed using SPSS version 21 (IBM Corp, Armonk, NY, USA).

\section{Results}

Of the 80 patients recruited in this study, 50 $(62.5 \%)$ were interpreted on ${ }^{18} \mathrm{~F}-\mathrm{FDG}$ PET/CT as having the same tumor, node, and metastasis (TNM) stage that was reported on conventional imaging, while 30 patients $(37.5 \%)$ had their AJCC stage changed according to the findings from ${ }^{18} \mathrm{~F}-\mathrm{FDG} \mathrm{PET} /$ CT. In the majority of them $(26 / 30 ; 32.5 \%$ of the total study cohort), ${ }^{18}$ F-FDG PET/CT detected new lesions, previously unseen by conventional imaging, leading to upstaging. On the other hand, the remaining four patients $(5 \%)$ were downstaged based on impressions from FDG PET/CT. There was a moderate agreement between conventional methods and ${ }^{18} \mathrm{~F}$ FDG-PET/CT in final TNM staging $(K=0.60)$.

Details of the TNM individual components are summarized in Table 1.

In 54 patients $(67.5 \%)$, both methods showed the same $\mathrm{T}$ status, while 26 patients $(32.5 \%)$ showed different local findings. ${ }^{18} \mathrm{~F}-\mathrm{FDG}$ PET/CT detected multifocal lesions (i.e., in different quadrants) more frequently ( $n=19,23.7 \%$ ) compared to the combination of mammography and ultrasonography $(n=8,10 \%)$.
Furthermore, ${ }^{18}$ F-FDG PET/CT detected multicentric lesions (i.e., within the same quadrant) in 10 patients $(12.5 \%)$ compared to four patients $(5 \%)$ detected by conventional methods.

Table 1: TNM staging

\begin{tabular}{llll}
\hline TNM & Conventional $(\mathrm{n} \%)$ & PET/CT $(\mathrm{n} \%)$ & Value of Kappa \\
\hline T1N0M0 & $1(1.3)$ & $0(0)$ & 0.60 \\
TON1M0 & $0(0)$ & $0(0)$ & \\
T1N1M0 & $3(3.8)$ & $0(0)$ & \\
T2N0M0 & $1(1.3)$ & $1(1.3)$ & \\
T2N1M0 & $6(7.5)$ & $2(2.5)$ & \\
T3N0M0 & $1(1.3)$ & $1(1.3)$ & \\
T3N1M0 & $8(10)$ & $10(12.5)$ & \\
TON2M0 & $0(0)$ & $0(0)$ & \\
T1N2M0 & $1(1.3)$ & $0(0)$ & \\
T2N2M0 & $2(2.5)$ & $2(2.5)$ & \\
T3N2M0 & $1(0)$ & $4(5)$ & \\
T4N0M0 & $1(1.3)$ & $2(2.5)$ & \\
T4N1M0 & $6(7.5)$ & $2(2.5)$ & \\
T4N2M0 & $5(6.3)$ & $3(3.8)$ & \\
Any T N3M0 & $5(6.3)$ & $11(13.8)$ & \\
Any T\&N M1 & $39(48.8)$ & $42(52.5)$ & \\
\hline TNM: Tumor, node, and metastasis. & & \\
& & &
\end{tabular}

${ }^{18}$ F-FDG PET/CT changed nodal status $(\mathrm{N})$ in 35 patients $(43.7 \%)$, mostly by upstaging $(n=28,35 \%)$ and less frequently by downstaging $(n=7,8.7 \%)$. ${ }^{18}$ F-FDG PET/CT revealed previously undetectable loco-regional lymphatic spread in infraclavicular ( $n=11 ; 13.7 \%)$, supraclavicular $(n=4 ; 5 \%)$, and internal mammary $(\mathrm{n}=12 ; 15 \%)$ nodal groups

${ }^{18}$ F-FDG PET/CT changed the distant status (M) in four patients (5\%) and three patients $(3.7 \%)$ were upstaged by detecting distant lesions (2 distant $\mathrm{LN}$ lesions and 1 bone lesion). While in one patient, ${ }^{18} \mathrm{~F}$ FDG-PET/CT excluded osseous deposits.

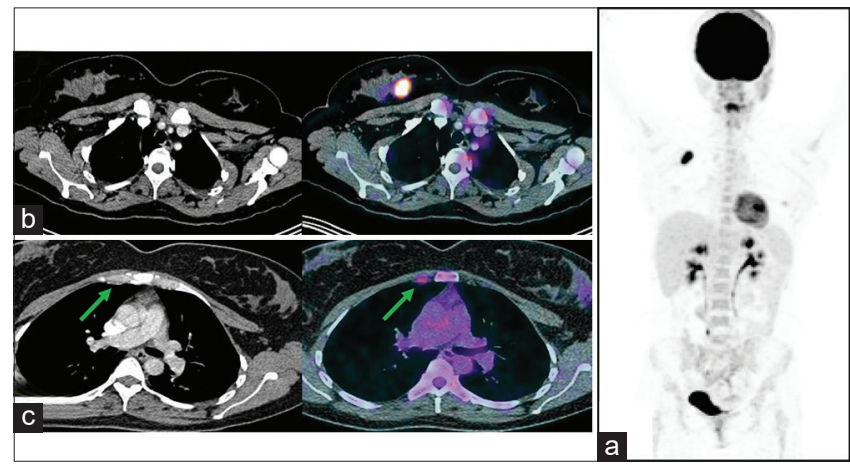

Figure 1: Three dimensional maximum intensity projection (a), axial CT and axial fused images for a 35-year-old female presented with right breast cancer; by conventional staging, the patient was stage T2N1M0 (stage IIB). Fluorodeoxyglucose (FDG) positron emission tomography/computed tomography (PET/CT) revealed metabolically active internal mammary lymph node that was indistinct in conventional images; the staging was upstaged to T2N3MO (IIIC). (b) FDG PET/CT showing metabolically active right breast neoplasm. (c) $P E T / C T$ showing metabolically active right internal mammary lymph node (green arrow)

\section{Extent of the disease}

Since the same AJCC stage can be determined by variable combinations of $\mathrm{T}, \mathrm{N}$, and $\mathrm{M}$ findings, not all differences in one component are reflected in a corresponding change in the overall AJCC stage. Although the same T, N, and/or M status can be assigned in many of these patients, details of the distribution and 
extent of the disease could have a major impact on management decisions.

${ }^{18}$ F-FDG PET/CT revealed previously unknown distant nodal involvement in additional 11 patients $(13.7 \%)$ by detecting FDG-avid lymph nodes in cervical, mediastinal, or abdominal nodes, all of them were not fitting the criteria for metastatic LNs on CE-CT.

${ }^{18} \mathrm{~F}-\mathrm{FDG}$ PET/CT revealed previously undetected osseous lesions in five additional patients $(6.2 \%)$ that were undetected by conventional imaging. ${ }^{18}$ F-FDG PET/CT and CE-CT were positive for an osseous spread in 24 patients. Of them, 14 patients showed more osseous lesions on ${ }^{18} \mathrm{~F}$-FDG PET/CT.

${ }^{18}$ F-FDG PET/CT revealed previously unknown hepatic deposits in two additional patients $(2.5 \%)$ that were undetected by conventional imaging.

Furthermore, ${ }^{18} \mathrm{~F}-\mathrm{FDG}$ PET/CT identified additional sites of distant disease in stage IV patients in 10 patients; pleura $(n=3)$, adrenal $(n=1)$, peritoneum $(n=1)$, adnexa $(n=1)$, pericardium $(n=1)$, and soft tissue $(n=2)$; all of them were missed on CE-CT.

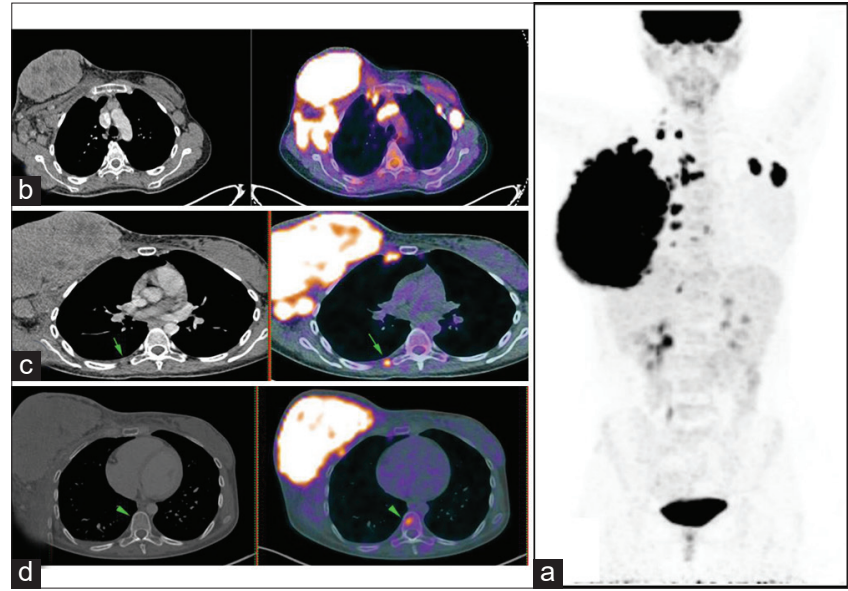

Figure 2: Three dimensional maximum intensity projection (a), axial CT and axial fused images for a 40-year-old female patient presented with right breast cancer; by conventional staging, the patient was stage T4N3M1 (Stage IV), showing multiple contralateral axillary and mediastinal nodes. After fluorodeoxyglucose (FDG) positron emission tomography/computed tomography (PET/CT), there was no change in staging, but FDG PET/CT reveals right pleural and osseous lesions that were indistinct in CT, (b) FDG PET/CT showed metabolically active locally advanced right breast cancer, metastatic axillary, and mediastinal lymph nodes, (c) FDG PET/CT showed metabolically active right pleural lesion (green arrow). (d) FDG PET/ CT showed a metabolically active osseous lesion in T9 vertebra (green arrowhead)

\section{Impact on management}

According to ${ }^{18}$ F-FDG PET/CT results, the radiation therapy field and/or systemic therapy was modified in 21 patients $(26.2 \%) .{ }^{18} \mathrm{~F}-F D G$ PET/CT led to extension of radiation field in 12 patients with internal mammary lymph nodal metastases (Figures 1-4).

Primary treatment approach was changed from surgery to neoadjuvant chemotherapy (before surgery)

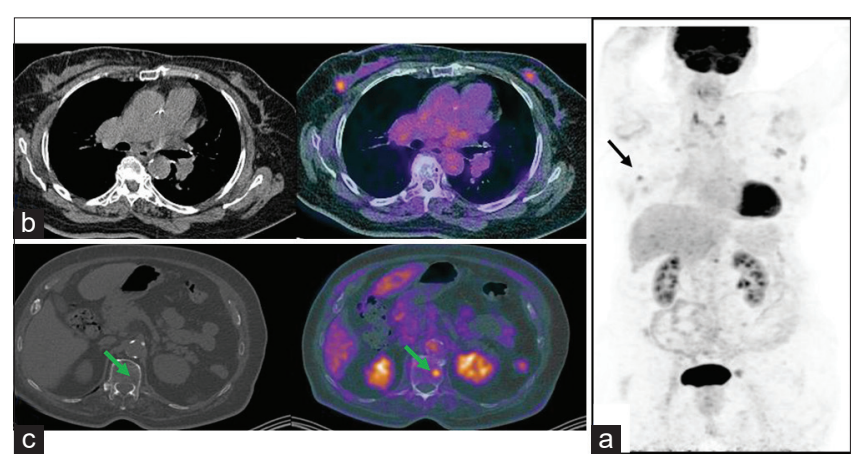

Figure 3: Three dimensional maximum intensity projection (a), axial CT and axial fused images for a 77-year-old female patient presented with left breast neoplasm; by conventional staging, the patient was stage T1N1M0 (stage IIA). Fluorodeoxyglucose positron emission tomography/computed tomography (PET/ CT) revealed another focal uptake corresponding to upper outer quadrant nodule in the right breast (black arrow) and metabolically active osseous lesions seen in iliac bones, T12, L5 vertebrae, and left acetabulum that were indistinct in conventional images; the staging was upstaged to T1N1M1 (Stage IV). (b) PET/CT showed metabolically active bilateral breast lesions. (c) PET/CT showed metabolically active $T 12$ vertebra that was indistinct in $C T$ images (green arrow)

in five patients, from curative to palliative treatment in three patients, and from palliative to curative treatment in one patient after 18F FDG-PET/CT excluded distant bony lesions that were considered positive on conventional imaging.

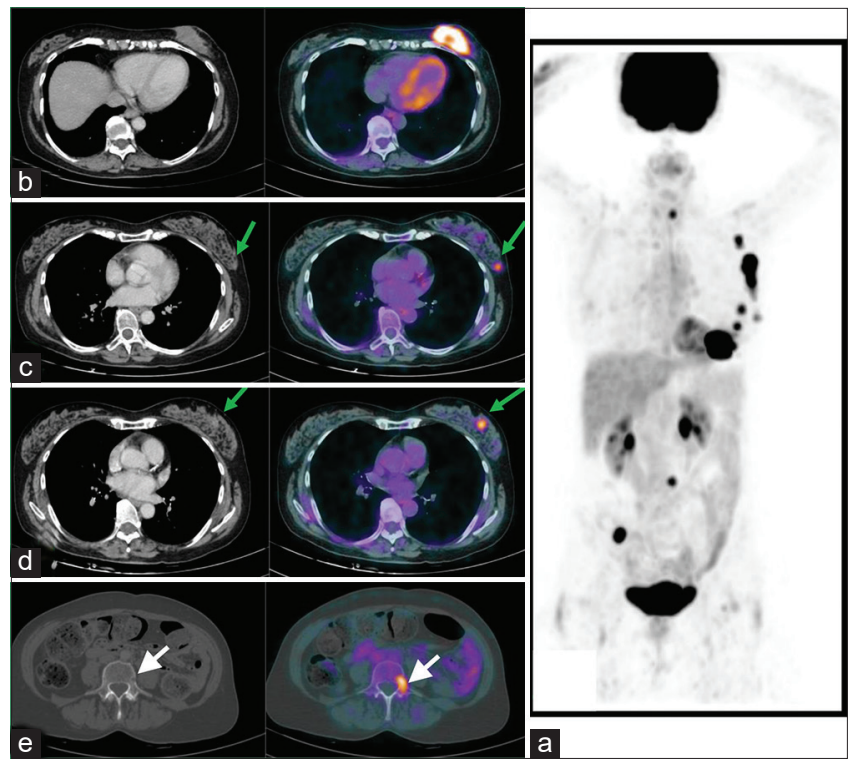

Figure 4: Three dimensional maximum intensity projection (a), axial CT and axial fused images for a 67-year-old female patient presented with left breast neoplasm; by conventional staging, the patient was stage T4N2M1 (stage IV) known to be metastatic to right iliac bone and T1 vertebra. After fluorodeoxyglucose (FDG) positron emission tomography/computed tomography (PET/CT), there was no change in staging, but FDG PET/CT revealed other breast nodules at different quadrants and osseous lesion in L4 vertebra that were indistinct in conventional imaging (b) showed metabolically active left breast neoplasm in the lower inner quadrant extending to the outer one and ulcerating to the overlying skin (c and d) showed metabolically active breast lesions in upper quadrants (green arrows) (e) showed a metabolically active osseous lesion in L4 vertebra that was indistinct in CT images (white arrows) 


\section{Discussion}

Breast cancer remains the most common type of non-skin cancer in women. Accurate staging is important for the management of the disease. In breast cancer staging, the TNM classification is used [10]. The National Comprehensive Cancer Network guidelines recommend assessment with ${ }^{18} \mathrm{~F}$ FDG PET/CT for staging of breast cancer, starting from the stage IIIA [11]. Our aim is to evaluate the contribution of ${ }^{18} \mathrm{~F}-\mathrm{FDG}$ PET/CT in the staging of breast cancer patients and the impact of this on the management approaches.

In this study, impressions from ${ }^{18} \mathrm{~F}$-FDG PET/ CT changed the AJCC stage in $30 \%$ of the patients. Similarly, Fuster et al. found that ${ }^{18} \mathrm{~F}-\mathrm{FDG}$ PET/CT led to a change in the initial staging in $42 \%$ of their cohort with 60 patients, reporting that the overall sensitivity and specificity for ${ }^{18}$ F-FDG PET/CT were significantly higher to those of diagnostic CT, which makes this technique recommendable in the staging of patients with large primary breast cancer. The results obtained in their study suggest that ${ }^{18} \mathrm{~F}-\mathrm{FDG}$ PET/ CT underestimates loco-regional lymph node staging in large primary breast cancer patients. Locally, 26 patients $(32.5 \%)$ of our cohort showed different local disease extent ( $T$ status) from ${ }^{18} \mathrm{~F}-\mathrm{FDG}$ PET/CT compared to conventional imaging. ${ }^{18} \mathrm{~F}-\mathrm{FDG}$ PET/CT detected multifocal lesions more frequently than the combination of mammography and ultrasonography. Although histopathological confirmation was not available, those patients were treated either with neoadjuvant chemotherapy or palliatively according to the final stage.

Gil-Rendo et al. published a prospective study focusing on the role of ${ }^{18} \mathrm{~F}-\mathrm{FDG}$ PET in the axillary staging of 275 patients with stage I and stage II diseases. The authors found a change of treatment in overall $33 \%$ of patients when taking into account sparing of sentinel lymph node biopsy in cases of positive FDG uptake in the axilla [12]. Likewise, in the current study, ${ }^{18} \mathrm{~F}$ FDG-PET/CT revealed previously undetectable regional lymphatic spread in a total of 27 patients (11 infraclavicular, 4 supraclavicular, and 12 internal mammary lymph nodes).

Bernsdorf et al. did not perform abdominal imaging or bone scans on any patients and their workup included only mammography, breast and axillary ultrasound, chest X-ray, and blood samples. In their study, ${ }^{18} \mathrm{~F}-\mathrm{FDG}$ PET/CT solely detected distant metastases (ovary, bones, and lung) in six patients and new primary cancers (ovary, lung) in another two patients. Furthermore, they reported 12 patients with extra-axillary lymph nodal involvement. In 15 patients (15\%), extra-axillary malignancy was detected by ${ }^{18}$ F-FDG PET/CT only, leading to an upgrade of initial staging in 14\% (14/103) and ultimately a modification of planned treatment in $8 \%$ $(8 / 103)$ of patients [13].

Similarly, in our study, ${ }^{18}$ F-FDG PET/CT detected additional sites of distant involvement in 10 patient ( 3 patients with pleural disease, 1 patient with adrenal deposits, 2 patients with peritoneal deposits, 1 patient with adnexal deposits, 1 patient with pericardial lesions, and 2 patients with soft tissue lesions) that were missed by conventional CT. ${ }^{18}$ F-FDG PET/ CT also revealed previously unknown distant extraaxillary metastases in $11 / 80$ cases $(13.7 \%)$ that were undetected by conventional CT.

In breast cancer, N3 nodal involvement (stage IIIC) has a poor prognosis [14]. Detection of extra-axillary lymph nodes may also have a major impact on the loco-regional treatment, for example, by defining the target volume for radiotherapy or the extent of the surgical clearance. Hence, ${ }^{18}$ F-FDG $\mathrm{PET} / \mathrm{CT}$ is a valuable tool to detect unsuspected extra-axillary lymph nodes and distant metastases [15]. According to our results, ${ }^{18}$ F-FDG PET/CT findings impacted treatment planning in $21(26.2 \%)$ patients, of them, 12 patients had their radiation field extended secondary to detecting internal mammary LN involvement on ${ }^{18}$ F-FDG PET/CT. Our results are in agreement with the results of other series [15], [16], [17].

The limitations of this study include a small sample size, which reduced the power of our conclusions, and the absence of histopathological validation. Many diagnostic studies fall short when it comes to the use of a real gold standard, especially when employing a whole-body coverage biopsy would be a questionable method, both from logistic and ethical perspectives. In practice, treating physicians usually make complex judgments decisions based on the best available evidence. We still believe that our findings are appropriate since the main purpose of this study was to answer the question of "detectability" and establish the magnitude of "differences" between the methods, rather than a "diagnostic performance" question, where an independent diagnostic method is required.

Another important limitation is that our patients were recruited in our study after a standard conventional workup was performed outside our institution; accordingly, the methodology employed in data acquisition and reporting quality cannot be verified.

On the other hand, the advantages of this study included its prospective design, uniform acquisition and interpretation protocols for the PET and CE-CT, and reporting the practical impact of $\mathrm{PET} / \mathrm{CT}$ reporting on the management of breast cancer patients with clinically suspected advanced local disease. We believe that our results can inform future hypothesis-driven prospective studies to study the impact of using one diagnostic tool versus the other. 


\section{Conclusions}

${ }^{18} \mathrm{~F}-\mathrm{FDG}$ PET/CT is an efficient imaging modality for staging of patients with clinically suspected advanced breast cancer not only for detecting distant lesions but also for detecting axillary and extra-axillary nodes and multifocality/multicentricity in the primary tumor site.

\section{References}

1. DeSantis CE, Fedewa SA, Sauer AG, Kramer JL, Smith RA Jemal A. Breast cancer statistics, 2015: Convergence of incidence rates between black and white women. CA Cancer J Clin 2016;66(1):31-42. https://doi.org/10.3322/caac.21320 PMid:26513636

2. Baba S, Isoda T, Maruoka Y, Kitamura Y, Sasaki M, Yoshida T, et al. Diagnostic and prognostic value of pretreatment SUV in 18F-FDG/PET in breast cancer: Comparison with apparent diffusion coefficient from diffusion-weighted MR imaging. J Nucl Med 2014;55(5):736-42. https://doi.org/10.2967/ jnumed.113.129395

PMid:24665089

3. Kurihara H, Shimizu C, Miyakita Y, Yoshida M, Hamada A, Kanayama $\mathrm{Y}$, et al. Molecular imaging using PET for breast cancer. Breast Cancer 2016;23(1):24-32.

PMid:25917108

4. Chikarmane S, Tirumani SH, Howard SA, Jagannathan JP, DiPiro PJ. Metastatic patterns of breast cancer subtypes: What radiologists should know in the era of personalized cancer medicine. Clin Radiol 2015;70(1):1-10. https://doi.org/10.1016/j. crad.2014.08.015

PMid:25300558

5. Yang HL, Liu T, Wang XM, Xu Y, Deng SM. Diagnosis of bone metastases: A meta-analysis comparing 18 FDG PET, CT, MRI and bone scintigraphy. Eur Radiol 2011;21(12):2604-17. https:// doi.org/10.1007/s00330-011-2221-4

PMid:21887484

6. Yamamoto $Y$, et al. Comparative analysis of imaging sensitivity of positron emission mammography and whole-body PET in relation to tumor size. Clin Nucl Med 2015;40(1):21-5. https:// doi.org/10.1097/rlu.0000000000000617

PMid:25423346

7. Boellaard R, Delgado-BoltonR, Oyen WJ, Giammarile F, Tatsch $\mathrm{K}$, et al. FDG PET/CT: EANM procedure guidelines for tumour imaging: Version 2.0. Eur J Nucl Med Mol Imaging 2015;42(2):328-54. https://doi.org/10.1007/s00259-010-1459-4 PMid:25452219

8. Abdelhafez Y, Tawakol A, Osama A, Hamada E, El-Refaei S. Role of 18F-FDG PET/CT in the detection of ovarian cancer recurrence in the setting of normal tumor markers. Egypt $J$ Radiol Nucl Med 2016;47(4):1787-94. https://doi.org/10.1016/j. ejrnm.2016.08.013

9. Rumsey DJ. Statistics II for Dummies. New York: John Wiley and Sons; 2009.

10. Akepati NK, Abubakar ZA, Bikkina P. Role of 18F-Fluorodeoxyglucose positron emission tomography/ computed tomography scan in castleman's disease. Indian J Nucl Med 2018;33(3):224-6. https://doi.org/10.4103/ijnm. ijnm_94_18 PMid:29962719

11. NCCN Clinical Practice Guidelines in Oncology (NCCN Guidelines): Breast Cancer-Version 3.2012. National Comprehensive Cancer Network. Available from: http:// www.nccn.org/professionals/physician gls/pdf/breast. pdf. [Last accessed on 2012 Nov 15]. https://doi. org/10.1111/j.1759-7714.2010.00016.x

12. Gil-Rendo A, Zornoza G, García-Velloso MJ, Regueira FM, Beorlegui C, Cervera M. Fluorodeoxyglucose positron emission tomography with sentinel lymph node biopsy for evaluation of axillary involvement in breast cancer. Br J Surg 2006;93(6):70712. https://doi.org/10.1002/bjs.5338 PMid: 16622900

13. Bernsdorf M, Berthelsen AK, Wielenga VT, Kroman N, Teilum D, Binderup $\mathrm{T}$, et al. Preoperative PET/CT in early-stage breast cancer. Ann Oncol 2012;23(9):2277-82

PMid:22357250

14. Edge SB, Byrd D, Compton CC, Fritz AG, Greene F, Trotti A AJCC Cancer Staging Manual. $7^{\text {th }}$ ed. New York: Springer; 2010.

15. Fuster D, Duch $J$, Paredes $P$, Velasco $M$, Muñoz $M$, Santamaría G, et al. Preoperative staging of large primary breast cancer with [18F] fluorodeoxyglucose positron emission tomography/computed tomography compared with conventional imaging procedures. J Clin Oncol 2008;26(29):4746-51. https:// doi.org/10.1200/jco.2008.17.1496 PMid:18695254

16. Segaert I, Mottaghy F, Ceyssens S, De Wever W, Stroobants S, Van Ongeval C, et al. Additional value of PET-CT in staging of clinical stage IIB and III breast cancer. Breast J 2010;16:617-24. https://doi.org/10.1111/j.1524-4741.2010.00987.x

17. Heusner TA, Kuemmel $S$, Umutlu $L$, Koeninger $A$ Freudenberg LS, Hauth EA, et al. Breast cancer staging in a single session: Whole-body PET/CT mammography. J Nucl Med 2008;49(8):1215-22. https://doi.org/10.2967/ jnumed.108.052050

PMid:18632831 